\title{
De l'éducation du corps à l'éducation au corps : genèse et critique de l'éducation en santé
}

\author{
Alexandre Klein Alexandre.Klein@univ-nancy2.fr
}

L'éducation pour la santé est un champ de recherche en crise, à tel point que par peur il se recroqueville dans ses bastions premiers, conservateurs, niant pour beaucoup les acquis des dernières années. Développée comme un champ spécifique des sciences de l'éducation par quelques précurseurs [1], l'éducation en santé [2] retrouve aujourd'hui, à l'occasion des réformes de santé et des nouvelles orientations institutionnelles de la santé publique en France, le champ médical duquel elle s'était difficilement extraite au cours de ces quinze dernières années pour s'affirmer comme pratique éducative à part entière. Les combats qu'elle avait menés pour son indépendance sont maintenant passés, ne reste que l'idée et l'instrumentalisation.

Dans ce contexte morose [3], il convient de s'interroger sur le type d'éducation en santé que nous souhaitons pour demain. Car si l'éducation en santé quitte le champ éducatif ne risque-t-elle pas également de s'éloigner des valeurs éducatives qui la fondent et ainsi de perdre si ce n'est son âme au moins l'essence de son domaine de réflexion et d'application ? Si l'éducation en santé déserte le champ des sciences humaines et sociales ne renie-t-elle pas le passé même qui l'a fondée ? Nous sommes conscients que cette contribution ne changera pas le destin institutionnel de l'éducation en santé, mais la réaffirmation de ses principes et la mise en évidence épistémologique de son champ d'action manifesteront (au sens fort du terme) les conditions de possibilités d'une éducation en santé qui respectent intégralement les notions qui la composent. Pour se vouloir, au sens plein du terme, éducation, l'éducation en santé ne peut se réduire à l'éducation thérapeutique, tout autant que pour traiter de santé, elle ne peut plus se restreindre au champ biomédical (Canguilhem, 1963 ; Klein, 2008 ; Klein, 2010a).

En épistémologue, nous nous proposons ici, dans la tradition française qui est la nôtre, de retracer l'historique de constitution de cette discipline, afin de cerner les enjeux de son apparition, et ce dans le but de définir les conditions de possibilité de son futur. L'idée n'étant pas d'attiser un débat en cours, mais de dépasser les champs d'opposition pour mieux les résoudre, à l'instar de la proposition philosophique de Stengers (1998) (comme peut-être de toute activité philosophique), nous penserons l'éducation en santé à partir et en vue des enjeux qu'elle implique sur le terrain des éducateurs autant que de notre société qui se souhaite démocratique. Nous choisirons donc l'analyseur qui s'impose ici pour dépasser et inclure les problématiques d'articulation de l'éducation et de la santé, autrement dit le corps, qui selon nous et nous le montrerons, permet de regarder sous un angle nouveau le problème posé tout en ouvrant les voies de sa résolution. Quelle définition du corps implique

\footnotetext{
1 Nous pensons ici à Georges Vigarello pour ses travaux fondateurs, mais également à Jacqueline Descarpentries qui avait mis en place l'un des seuls masters d'éducation et santé en sciences humaines et sociales sur le territoire français au sein de l'UFR de Sciences de l'éducation de l'université Lille 3.

2 Nous entendons sous ce vocable toutes les tentatives d'articulation de la santé et de l'éducation que ce soit pour ou à.

3 L'absence de reconnaissance du statut professionnel d'éducateur en santé, la disparition progressive des formations à la santé en sciences de l'éducation, la reprise en main par le monde médical de ce champ de recherche par le biais de l'éducation thérapeutique du patient en sont quelques symptômes.
} 
l'éducation en santé ? Quel usage du corps organise-t-elle ? Et en conséquence, quelle éducation voulons-nous pour respecter le corps que nous avons, que nous sommes et que nous souhaitons? A partir des travaux de Michel Foucault (1926-1984), qui organisèrent l'apparition de la discipline éducation pour la santé et enrichirent notre propre réflexion, nous tenterons d'éviter les écueils d'une éducation normalisante du corps pour retrouver une éducation en santé digne des valeurs d'éducation et de santé (Klein, 2010b). C'est ainsi que nous nous dirigerons vers une éducation au corps, nouvelle modalité d'articulation de l'éducation et de la santé, qui en respecte les valeurs et objectifs autant qu'elle en renouvelle les définitions.

\section{Santé publique et éducation}

La santé publique étant définie de manières multiples, nous admettrons dans un premier temps la définition conventionnellement admise par tous de Tessier : « une approche collective et administrative des problèmes de santé des populations sous ses aspects politiques, économiques, réglementaires, institutionnels » (Tessier et al., 1996, p. 29). La santé publique vise donc, par une organisation des problématiques de santé des populations, une amélioration de cette dernière magnifiée par la réduction du taux de mortalité et l'augmentation de l'espérance de vie. En retraçant sa genèse on s'aperçoit que ce n'est qu'en dernier lieu qu'elle fit appel à l'éducation.

En effet, c'est d'abord comme hygiène publique que l'on s'est intéressé à la santé des populations. Craignant la diminution démographique de leurs populations, les gouvernants des pays européens, dès le XVII ${ }^{\mathrm{e}}$ siècle, mettent en place une organisation et une gestion politique des conditions de vie des individus dans leur milieu. C'est le temps de la police médicale (Frank, 1779-1819), de la sanitarisation des villes, de la purification de l'air, de l'assèchement des marais, bref de l'organisation écologique des conditions d'existence des populations. L'hygiène publique se veut en rupture avec l'hygiène hippocratique (Barroux, 2008), c'est-à-dire un ensemble de règles de vie, et s'intéresse donc peu aux existences individuelles. Son but est de lutter contre les maladies infectieuses, les épidémies qui ravagent alors les territoires. La vaccination inaugurée par Edward Jenner (1749-1823) marque alors parfaitement cet intérêt pour les populations. Mais rapidement, cette gestion de l'espace montre ses limites et, en 1854, et ce malgré les efforts consentis en matière d'aménagement du territoire depuis l'épidémie de 1832 (Delaporte, 1990), le choléra ravage la France. Le modèle public et écologique montre ses limites et en l'absence de manœuvres thérapeutiques et préventives efficaces (qui n'arriveront qu'avec Louis Pasteur (1822-195) et la sérologie), il faut changer de stratégie.

On renoue alors avec l'idée antique d'hygiène comme mode de vie en mettant l'accent sur la nécessité de transmettre aux individus les règles élémentaires d'hygiène qui ont été mis en lumière. Ainsi, si l'antisepsie, introduite en France en 1874 par le docteur Just LucasChampionnière (1843-1913), qui s'était formé auprès du chirurgien britannique Joseph Lister (1827-1912), est rapidement adoptée par les médecins puis par les élites éclairées, au moyen des traités de vulgarisation médicaux, il faudra plusieurs décennies pour que la population dans son ensemble accepte l'idée d'un lavage des mains régulier.

Certes, déjà, les tracts, affiches, brochures, campagnes de presse, exposition ou dessins tentent de répandre les préceptes de l'hygiène dans les populations, mais l'impact est encore faible. Car les populations visées, avant tout pauvres, étaient souvent analphabètes. Il fallait 
donc trouver un lieu de propagande (au sens non péjoratif du terme) des préceptes hygiénistes. Ce sera l'école, désormais obligatoire depuis les lois Ferry de 1881-1882.

L'idée générale de cette éducation sanitaire était d'inculquer, principalement aux classes les plus défavorisées, les normes d'un monde bourgeois plus sanitarisé afin de favoriser la santé générale de la population et donc la sécurité sanitaire de ces classes privilégiées. Il s'agissait en grande partie de faire peur aux populations en insistant sur le risque de la maladie et de la mort. Il fallait éduquer le corps à des normes spécifiques afin de valoriser la santé de la population dans laquelle s'inscrivait l'individu. En accord avec la doctrine en vogue au XIX ${ }^{\mathrm{e}}$ siècle, on souhaitait de favoriser les causes de la bonne santé chez les populations afin de lutter contre le risque interne à la société de dégénérescence. A l'instar du discours succédant à la défaite de 1870, les populations devaient alors se mobiliser au profit de la Nation en suivant les recommandations des médecins dont l'autorité reposait sur la certitude d'un savoir scientifique. L'éducation était alors mise au service de la santé publique, mais moins comme un outil spécifique, que comme un lieu d'expression. Les instituteurs sont donc, dès la seconde moitié du XIX ${ }^{\mathrm{e}}$ siècle intégrés aux dispositifs d'hygiène public. Leur rôle premier d'éducation et d'instruction s'étend alors à la surveillance et à la diffusion des règles d'hygiène. "L'instituteur et l'institutrice doivent alors vérifier et promouvoir la propreté corporelle, enseigner la gymnastique, convaincre les familles de la légitimité de la vaccination antivariolique et des méfaits de l'alcool [...] Dorénavant, ils auront aussi à charge le renforcement des corps (par la gymnastique par exemple) et la préservation contre les déviances physiques, intellectuelles et morales » (Parayre, 2009). L'objectif réside alors plus dans une sauvegarde collective de la population et une normalisation généralisée des comportements sanitaires par l'école. De l'organisation sanitaire des locaux pour lutter contre les épidémies, on glisse doucement vers l'exigence de soins individuels du corps. Des locaux au corps de l'élève, la santé publique ne change pas de modèle et demande une réorganisation sanitaire des usages que l'on fait des espaces (école, hôpitaux, etc.) comme des étendues (les corps).

Cette action de normalisation des comportements individuels au profit de la santé de la population, qui se concrétise avec l'entrée en scène de l'éducation dans le dispositif de santé publique, marque également l'avènement de la santé publique comme un biopouvoir disciplinaire - pour reprendre les termes du philosophe Michel Foucault - autrement dit, comme un dispositif de pouvoir déterminant la liberté des individus. Avec l'éducation sanitaire, la santé publique concrétise son projet de gestion par la normalisation des usages du corps. L'éducation comme dispositif disciplinaire achevait de clore le modèle biopolitique de la santé publique, ouvrant, dès lors, le paradoxe qui allait animer le $\mathrm{XX}^{\mathrm{e}}$ siècle et qui nous pose aujourd'hui problème.

\section{La santé publique comme biopouvoir : La normalisation des usages du corps}

Pour comprendre cet état de fait, il nous faut revenir sur la théorie foucaldienne de la biopolitique. En analysant la naissance de la prison, dans son célèbre ouvrage Surveiller et punir, Foucault (1975) note que notre modernité s'est constituée par la substitution d'un pouvoir disciplinaire à un pouvoir souverain. Le premier, identifié dans la personne du souverain, vertical puisque fonctionnant par l'application spectaculaire de la loi dans la cérémonie du supplice, se résumant dans la maxime « laisser vivre et faire mourir », laissait progressivement au cours du XVIII ${ }^{\mathrm{e}}$ siècle sa place à un pouvoir tout différent, anonyme et horizontal puisque relayé au moyen des normes par tout un chacun, et pouvant se résumer dans la maxime «faire vivre et laisser mourir ». Là où le pouvoir souverain marquait le corps 
des individus de sa puissance par la violence des supplices ou de la question, le pouvoir disciplinaire visait la normalisation des usages du corps par des moyens moins violents telles la gestion de l'existence par l'emploi du temps ou l'organisation des espaces de vie. Avec la discipline, le corps se retrouvait parcouru de faisceaux de pouvoir qui le contraignaient à un certain mode d'existence, à certains comportements, favorisant ainsi son individualisation. Cette gestion politique des corps, cette anatomopolitique de la discipline allait très vite, sous l'influence du libéralisme (Foucault, 2004) naissant, faire le jeu d'une autre forme de pouvoir, dit biopolitique, c'est-à-dire s'appliquant non plus aux individus mais à la population comprise comme un ensemble d'individus régis par des lois biologiques communes. La biopolitique est la gestion politique de la vie des populations au profit de son accroissement et de sa régulation, elle fonctionne au moyen de différents biopouvoirs, dont la santé publique par exemple. On l'a vu, les directives de l'hygiène publique, par l'assainissement des villes ou l'organisation de la vaccination massive, s'inscrivent dans cette perspective de gestion de la population en vue de l'accroissement de sa santé et de la régulation de son développement. Mais ce pouvoir biopolitique ne prend vraiment forme que lorsqu'il retrouve le pouvoir disciplinaire à partir duquel il s'est développé [4]. Car il y a bien clôture du système de pouvoir biopolitique lorsque la gestion des existences individuelles (discipline) se fait au profit de l'accroissement et de la régulation de la vie de la population (biopolitique). Or, c'est bien ce que réalise l'éducation sanitaire, la clôture du modèle biopolitique par l'association de procédés disciplinaires mais en vue d'accroitre le biopouvoir de la santé publique.

C'est en ce sens que Foucault, mais également Illich (1975) ou Massé (2003) ont dénoncé la santé publique comme une entreprise de normalisation des existences individuelles au profit de l'accroissement et de la régulation de la vie de la population à laquelle ils appartiennent. De l'hygiène à la sexualité en passant par la santé, ce sont toutes les données de la vie biologique de la population qui sont prises en charge par le pouvoir politique, aux moyens de biopouvoirs scientifiques, techniques ou disciplinaires, afin d'accroitre l'espérance de vie et de réduire le taux de mortalité, menant ainsi à une unification des usages du corps, des comportements, des existences. La santé publique s'est constituée comme une culture à part entière qu'il s'agit d'adopter afin de favoriser sa santé et celle de la population qui nous accueille.

Ainsi, de la gestion géographique ou écologique des conditions de vie des populations, la biopolitique va renouer, par le truchement de l'éducation, avec la normalisation des usages du corps des individus, dans la droite ligne de l'anatomopolitique disciplinaire comme application aux détails du corps individuel et de ses usages des règles médico-scientifiques de l'hygiène. Les techniques disciplinaires, modifiées par la biopolitique, servent désormais les intérêts régulateurs de cette nouvelle technologie de pouvoir et ne favorisent plus l'individuation des sujets. L'enjeu biopolitique a annihilé la puissance individualisante de la discipline, tendant ainsi à aliéner la liberté des sujets (Klein, 2010b). La lutte contre l'onanisme ou même l'usage du vélo par les femmes à la fin du XIX ${ }^{\mathrm{e}}$ siècle en sont de parfaits exemples. Ainsi, parce que l'on croit que la masturbation affaiblit l'esprit autant que le corps, la sexualité des enfants est très rapidement prise en charge à tous les niveaux de la société : les médecins établissent la vérité de ces comportements en les qualifiant de déviants, puis ce savoir «scientifique » est transmis à la famille et à l'école

\footnotetext{
4 Si la discipline se met en place au XVII ${ }^{\mathrm{e}}$ siècle et au cours du XVIII ${ }^{\mathrm{e}}$ siècle, une nouvelle technologie du pouvoir se fait jour au XVIII ${ }^{\mathrm{e}}$ qui ne remplace pas la discipline mais en déplace l'action. Une technologie du pouvoir qui « l'emboite, qui l'intègre, qui la modifie partiellement et qui surtout, va l'utiliser en s'implantant en quelque sorte en elle, et s'incrustant effectivement grâce à cette technique disciplinaire préalable », (Foucault, 1997, p. 216).
} 
assorti d'une demande de contrôle. Ainsi, la lutte contre la masturbation, qui est considérée comme affaiblissant les individus et donc la nation puisque cela contrevient à la natalité (notamment depuis Tissot, 1760), s'impose dans les usages du corps, à tel point qu'en 1880, certains chirurgiens pratiquent encore l'ablation du clitoris des fillettes se masturbant. Le corps est modelé, par l'éducation et in extremis par la chirurgie, selon des normes ayant trait à la population et à son développement. De même, Bohuon (inédit) a montré que la bicyclette ou l'équitation seront, un temps, interdites médicalement aux femmes puisque ces pratiques physiques sont jugées pathogènes. L'atteinte des organes génitaux et donc reproducteurs est alors impensables puisqu'il est du rôle de la femme d'enfanter (O'Followell, 1900, p. 8), tant dans sa nature même que vis-à-vis de son rôle national, populationnel. Sous couvert du bienêtre et de l'accroissement de la population, les comportements individuels et les usages du corps par l'individu sont gérés et normalisés jusqu'à l'extrémisme de la normalisation physique par la chirurgie.

L'éducation, fleuron de la discipline, est réinvestie à des fins biopolitiques, elle devient alors le vecteur premier de la transmission des normes biopolitiques auprès des individus. Elle est un média instrumentalisé au profit de considérations qui lui sont extérieures. Et finalement elle sert le projet de normalisation des existences individuelles au profit d'une norme populationnelle, s'imposant dès lors à l'inverse de ce qu'elle revendique être, c'est-àdire un moyen de développement et d'épanouissement de l'individu comme être autonome et libre [5]. Ce paradoxe apparaîtra avec force [6] dans l'avènement du régime nazi qui, mettant en place au pied de la lettre le projet biopolitique, finira par exterminer tous ceux qui ne participent pas à l'accroissement de la race aryenne. Mais c'est finalement moins le risque biopolitique que certaines victoires contre les maladies infectieuses grâce notamment aux découvertes thérapeutiques comme les antibiotiques ou résultat des campagnes de vaccination, qui engagèrent le changement de perspective et la rupture avec l'éducation sanitaire.

\section{L'éducation à la santé une volonté de renouveau}

\section{De l'éducation sanitaire à l'éducation pour la santé}

La réduction des risques des maladies infectieuses orienta le regard de la santé publique vers les maladies chroniques dégénératives comme le cancer ou les maladies cardiovasculaires qui devinrent, à la suite des années 1950, les nouvelles cibles de la médecine préventive. Or ces maladies impliquaient non plus une simple information, mais un véritable changement de mode de vie de la part des individus. Il s'agissait désormais d'éviter les comportements à risques. Cette réorientation des objectifs de prévention coïncida avec l'apparition et le développement des médias de masse et cette coïncidence favorisa le développement d'une prévention nouvelle, rapidement institutionnalisée dans les années 1970 en Europe comme aux Etats-Unis : l'éducation pour la santé. De plus, et comme le montre

\footnotetext{
5 Le Trésor de la Langue Française indique comme définition de l'éducation : «Art de former une personne, spécialement un enfant ou un adolescent, en développant ses qualités physiques, intellectuelles et morales, de façon à lui permettre d'affronter sa vie personnelle et sociale avec une personnalité suffisamment épanouie ». http://atilf.atilf.fr/dendien/scripts/tlfiv5/visusel.exe?32;s=3375775680;r=2;nat=;sol=1; [consulté le 15 septembre 2009]

6 Apogée d'une idéologie développée au $\mathrm{XIX}^{\mathrm{e}}$ siècle, le mouvement nazi réalisera des actions d'eugénisme négatif en éliminant les populations jugées non conformes à leur idéal raciste, mais également des actions d'eugénisme positif en favorisant, notamment par le biais de l'éducation au corps et à la santé, le développement des meilleurs éléments de la race aryenne. Voir à ce propos, Pichot, 2000 ou Gayon et Jacobi, 2006.
} 
Berlivet (2004) dont nous allons suivre la réflexion sur la généalogie de l'éducation pour la santé en France, cette nouvelle modalité d'articulation de l'éducation et de la santé s'est constituée dans un nouveau paradigme, psychosocial, rejetant l'autoritarisme et le paternalisme biomédical incarnés par l'éducation sanitaire. Riche des travaux de Herzlich (1969) sur le modèle psychosocial de la santé, de Szasz (1975) sur la maladie mentale, de Foucault (1975) sur le pouvoir disciplinaire, d'Illich (1975) sur l'aspect pathogène de la médecine, l'éducation pour la santé avait pour ambition de contredire la tendance totalitaire de la santé publique en s'inspirant de ces modèles critiques du modèle médical. Elle reprenait à son compte la définition de la santé donnée par l'OMS dans le préambule de sa constitution en 1946 [7].

C'est ainsi que sous l'influence de Simone Veil, alors Ministre de la Santé, ellemême conseillée par la commission Cancer, la France se dota en janvier 1976 d'une loi qui visait la limitation de la publicité sur le tabac et l'interdiction du tabac dans certains lieux publics. A ce volet législatif s'ajoutait une volonté de créer une vaste campagne d'informations sur les dangers du tabac. Suite à ce premier essor politique et législatif, une institution spécifique, à la fois spécialisée et à l'organisation unique [8], fut mise en place : le Comité Français d'Education pour la Santé (CFES). Cette nouvelle institution favorisa le développement d'un savoir nouveau de la prévention, grandement fondé sur les sciences humaines et sociales, puisqu'elle avait pour contrainte d'être indépendante de l'administration de la santé et de ne pouvoir tisser aucun lien avec les médecins de la santé publique ni même avec les entrepreneurs associatifs de ce milieu.

Pour des raisons aussi institutionnelles que théoriques donc, on s'évertua à rompre avec les procédés biopolitiques de l'éducation sanitaire, valorisant une définition positive de la santé, éloignant l'outil de la peur de la maladie. Le Comité avait alors pour but d'aider les Français à trouver la motivation nécessaire pour se libérer des pratiques à risques résultantes d'un stress dû aux conditions de vie en société. On renversait donc la perspective, le bien-être des individus primait sur le renouvellement de la société pathogène. Il ne s'agissait plus de blâmer la victime mais de susciter et d'entretenir des résistances à l'influence néfastes de la société. L'accent était alors mis sur l'autonomisation des individus plutôt que sur leur normalisation. Les recrutements massifs du CFES qui se déroulèrent dans la seconde moitié des années 1970 accentueront ce virage puisque les nouveaux membres étaient loin des anciens professeurs d'hygiène et peu d'entre eux étaient médecins.

Tout semblait donc aller vers le mieux. Le déploiement des modalités d'épanouissement des individus ciblés par l'éducation pour la santé remplaçait la normalisation biopolitique des comportements développés par l'éducation sanitaire.

\section{De la normalisation des corps à l'impératif de leur soin.}

Dès la fin des années 70, les actions de prévention du CFES se sophistiquèrent, articulant les travaux de psychosociologues au savoir-faire expert de publicitaires et de cinéastes. "L'ambition avouée était désormais de développer une véritable ingénierie symbolique » (Berlivet, 2004, p. 55). Il s'agissait de renverser la charge symbolique de certains comportements considérés à risques, comme l'usage du tabac ou de l'alcool, qui

7 L'OMS dans le préambule de sa constitution de 1946 définit la santé comme : « un état de complet bien-être physique, mental et social, et ne consiste pas seulement en une absence de maladie ou d'infirmité ».

8 Bien que régi par la loi de 1901 et créé comme une association, le CFES était néanmoins dirigé par un « délégué général à l'éducation pour la santé » et un président de conseil d'administration agréés par le ministère. 
servaient alors d'outils de socialisation pour les jeunes. L'ambition était bien de donner une nouvelle culture à la santé en transformant les schémas sociaux habituellement acquis et diffusés. La cigarette ne devait plus être considérée comme «cool» par les jeunes. De l'information sur les risques, on passait alors aux processus d'individuation, par une sorte de retour vers le modèle disciplinaire : l'idée était de favoriser la conscience de soi des individus ciblés pour favoriser leur résistance aux modèles largement diffusés. En un certain sens, on entrainait les individus à faire acte de rébellion, de résistance face aux images sociales positives diffusées par les publicitaires des marques d'alcool et de cigarettes, pour ne prendre que ces deux exemples. On insistait alors sur la prise de conscience de la normalisation subie dans l'environnement social, afin d'engager un rejet de ces modèles qui favorisaient les comportements à risques, et ce dans le but, de s'affirmer en tant qu'individu. Les apports du psychosociologue Emeric Deutsch (Berlivet, 2004, p. 59) aidèrent à comprendre les motivations des individus et à forger des concepts comme le mythe du fumeur ou l'image sociale de la cigarette. On décryptait les comportements humains et leur mise en place afin de mieux combattre les comportements à risques et favoriser les comportements de santé.

Très vite, les campagnes de prévention valorisèrent l'abstention plutôt que la modération et l'image renvoyée par ces campagnes ne fit pas long feu face à l'image positive des fumeurs et des buveurs de la publicité ou du cinéma. Si l'on avait compris que l'on pouvait devenir un homme sans pour autant fumer, la figure de ce non-fumeur ne donnait alors pas envie. On s'évertua donc à construire une image positive et valorisante du nonfumeur affranchi de cette dépendance.

Mais de l'autocontrôle disciplinaire à la normalisation biopolitique, il n'y a avait qu'un pas que les campagnes de prévention franchirent sans même s'en rendre compte. En voulant fuir l'éducation normalisante du corps, on avait finalement rejoint la normalisation des esprits. Et les populations n'étaient pas dupes, puisque les résultats n'étaient pas au rendez-vous. In fine on s'orienta vers la diffusion de messages visant l'internalisation de l'exigence de contrôle. L'extériorité de ces messages était en effet rejetée comme normalisante par les populations. On visa donc le développement de l'estime de soi et de la confiance en soi. Mais la demande sous-jacente ne disparut pas pour autant et ces campagnes participèrent d'un renforcement de l'internalisation de la norme. Si l'épanouissement était là, il n'en était pas, pour autant, moins orienté. Car il serait naïf de croire que la simple adoption d'un mode de vie où l'individu se réalise pleinement coïncide avec un mode de vie sans comportements à risques.

\section{Deux modèles pour une même normalisation : un oubli du corps vécu}

On constate que, malgré les bonnes volontés et les différents modèles, l'association entre éducation et santé tend toujours à une forme de normalisation niant le sujet dans son individualité vécue (Klein, 2010b). Si une même normalisation a lieu dans les deux modèles d'éducation en santé que sont l'éducation sanitaire et l'éducation pour la santé, c'est que le corps y est toujours considéré comme un matériau à modeler et à gérer par l'individu selon des considérations populationnelles. C'est le problème de la volonté qui est en fait posé, à nouveaux frais par l'éducation pour la santé. Puisqu'il est indigne de normaliser les corps, on normalise les styles de vie afin d'atteindre le même résultat.

Malgré toute sa bonne volonté, l'éducation pour la santé reste enfermée dans des considérations biopolitiques à l'encontre desquelles elle s'est pourtant construite. Car la santé comme l'éducation reposent sur une multiplicité de modèles et demandent une réflexion 
épistémologique poussée pour en résoudre les articulations. Des travaux dans ce sens ont été réalisés, mais en restent, indépendamment de leur grande rigueur et intérêt, à leur balbutiement du fait même de leur approche interdisciplinaire qui développe une strate supplémentaire à la problématique épistémologique déjà complexe de l'éducation et de la santé.

Il nous semble que pour éviter les écueils d'une épistémologie encore en construction, tout en participant activement à sa consolidation, il faille adopter un tiers pour penser cette relation. Il est du rôle de l'activité philosophique de proposer des voies d'accès nouvelles pour penser des problématiques embourbées dans leur mise en forme. La philosophie du corps (Bruaire, 1968; Andrieu, 2001; Andrieu, 2009) participe ici de ce décentrement nécessaire au regard du philosophe. En explicitant le corps comme un analyseur (Brohm, 2001), elle nous offre en effet un outil parfaitement adéquat aux domaines en jeu dans la problématique, puisque de l'éducation sanitaire à l'éducation pour la santé, le statut du corps est mobilisé autant que questionné. La normalisation des usages d'un corps biologiquement et médicalement défini comme l'appel à l'autocontrôle d'un corps soumis à notre volonté à son utilisation, dévoilent un même rapport au corps, une même articulation de la vie du sujet à son corps. Le corps se situe même comme un au-delà du débat en questionnant les fondements mêmes - au-delà de la forme - de l'interaction entre éducation et santé. La santé est-elle une donnée à partir de laquelle on peut envisager de vivre avec son corps, de se vivre en tant que sujet corporel ? La description épistémologique des conditions de possibilité normatives [9] du rapport vécu à son corps, bref des conditions de possibilité de la subjectivation corporelle, nous indique la voie d'une solution au problème de l'éducation en santé. Comment vivre son corps de manière telle que ce vécu participe à la constante subjectivation normative de l'individu? La réponse à cette question est essentielle à la résolution épistémologique autant que pratique de l'éducation en santé.

Pour ce faire, il nous faut poursuivre l'analyse des travaux de Michel Foucault qui avait, en toute continuité avec ses œuvres précédentes [10], offert les moyens de résolution de la problématique biopolitique dans les ouvrages de sa dernière année d'existence (Foucault, 1984a, 1984b). Foucault avait fait son épistémè [11], avec la mise en évidence de la biopolitique, mais il avait également permis son dépassement dans sa généalogie ontologique (Han, 1998) du sujet corporel (Andrieu, 2004).

\footnotetext{
$9 \mathrm{Au}$ sens ici de la normativité et non de normes comme un excès de langage actuel le fait trop souvent comprendre.

10 On considère habituellement qu'il y a un Foucault de l'archéologie (1960-1970), de la généalogie (19701980), puis de la subjectivation (1981-1984), la méconnaissance des cours au Collège de France de Foucault entre la parution du premier tome de l'histoire de la sexualité (1976) et les deux derniers (1984), a participé à l'idée d'une rupture foucaldienne qu'alimentait la surprise du changement de point de vue et d'époque des deux derniers volumes de son Histoire de la sexualité par rapport au premier et au programme qu'il y annonçait. Mais cette opinion n'est plus tenable, surtout depuis la publication des cours au Collège de France où se dévoile la mutation de la pensée de Foucault. Nous nous accordons donc avec l'idée d'une continuité de la pensée foucaldienne telle que défendue par Revel (2004).

11 L'épistémè est pour Foucault l'ensemble des questions posées et des réponses données à certaines questions à une époque donnée au sein d'une culture spécifique. Sans nier les différences conceptuelles majeures, cette notion se rapproche de celle de paradigme développée par Kuhn (1962).
} 


\section{Le sujet comme limite mais non comme assurance : le gouvernement de soi et des autres}

Dans le troisième mouvement de son œuvre, Michel Foucault a déplacé son regard des savoirs et des pouvoirs vers les rapports du sujet à ses instances. La mise en évidence des processus de subjectivation /objectivation qui participent à la constitution de l'identité subjective de l'individu a permis à Foucault de relire son œuvre à l'aune d'une histoire des modes de constitution du sujet corporel. Or, cette perspective du sujet, qui impose une relecture de la normalisation biopolitique, ouvre également les voies de sa résolution. Ce que montre Foucault c'est qu'au-delà des normes qui s'imposent au sujet ou que celui-ci s'impose, il y a toujours un «jeu du je » par rapport à ces normes. L'objectivation du sujet qu'imposent les normes est coextensive d'une subjectivation qui peut prendre des formes différentes allant de la normation [12] pure à la normativité [13] en passant par la normalisation. Autrement dit, l'important n'est pas tant les normes, qui n'ont aucun pouvoir en elles-mêmes, que ce que le sujet fait de ces normes. Le risque biopolitique n'est donc plus dans l'uniformisation des comportements au profit du pouvoir et de l'argent, mais dans l'impossibilité de jouer avec les normes, de faire acte de normativité. La biopolitique est toxique lorsqu'elle interdit la création de nouvelles normes. La normalisation sociale est totale lorsque la normativité est rejetée en dehors du cercle de la société (Le Blanc, 2002, p. 238) [14]. Mais en dehors de ce cas extrême, le sujet peut toujours faire preuve de liberté vis-à-vis des normes tant qu'il préserve sa capacité première de transgression des normes. La liberté est la possibilité de transgression des normes, elle est une forme de résistance (Foucault, 2001, p. 1539). Comme le résume Veyne, «Nous ne pouvons échapper nulle part aux relations de pouvoir; en revanche, nous pouvons toujours et partout les modifier; car le pouvoir est une relation bilatérale ; il fait couple avec l'obéissance, que nous sommes libres (oui, libres) d'accorder avec plus ou moins de résistance. » (Veyne, 2008, p. 143).

Dans ce travail sur la subjectivation, l'important n'est donc plus le pouvoir, mais la gouvernementalité, c'est-à-dire la manière dont les sujets se rapportent au pouvoir. Foucault montre ainsi qu'il est possible de maintenir un gouvernement de soi qui reste éthique, c'est-àdire incluant la liberté du type de la normativité, et ce malgré la normalisation galopante de la société moderne. Il développe ainsi un modèle d'accompagnement éducatif permettant le maintien de la liberté des individus ciblés. Le «bon » gouvernement des autres est celui qui implique et maintient le gouvernement de soi par soi (Foucault, 2008). Il s'agit à la fois de conserver chez l'accompagné un gouvernement de lui-même par lui-même, que l'on nommerait aujourd'hui autonomie, mais plus, le gouvernement des autres, le travail de l'accompagnant, doit avoir pour unique objectif le développement et le maintien du gouvernement de soi. Le sujet en construction vers l'affirmation de sa liberté, autrement dit la subjectivation éthique, est la norme de toute éducation, et plus encore de l'éducation en santé.

Dans cette veine, il est donc possible de développer des actions d'éducation en santé qui ne participent pas de la normalisation biopolitique des corps et des individus. L'intégration des populations à la construction des programmes de prévention en est le premier vecteur. A l'instar de ce qu'a pu développer le Canada autour de la santé

12 La normation est définie par Legrand (2007) comme l'imposition coercitive de dispositions durables correspondant à la discipline.

13 La normativité est définie comme capacité de création de nouvelles normes (Canguilhem, 1963)

$14 \mathrm{La}$ normalisation sera complètement effective lorsque nous assisterons à un «recouvrement tel de la normativité par la normalisation que la normativité sociale ne peut plus s'exercer dans la normalisation ». 
communautaire, il faut une communauté d'acteurs pour construire un accompagnement qui remettent en question les notions même d'accompagné et d'accompagnant. Dedeine (2009) a montré comment, au sein d'une structure associative OSMOSE, l'accompagnement des personnes ayant une problématique de santé mentale pouvait être constitué par ses membres eux-mêmes. Acteurs de la structure du comité d'administration à l'établissement des propositions d'activités, les accompagnés deviennent accompagnants, ce qui favorisent leur subjectivation comme personnes à part entière et non comme malades mentaux. Plus que de favoriser leur autonomie ou leur empowerment, cette initiative de santé communautaire prend au pied de la lettre les indications foucaldiennes d'un gouvernement des autres modelé «par et pour » vers le gouvernement de soi. Les membres en besoin d'accompagnement trouvent toujours des professionnels ou des accompagnés ayant pratiqué le gouvernement de soi, pour être amenés à se soucier d'eux-mêmes et ainsi à développer, par le biais de l'estime et de la confiance en soi, un véritable gouvernement d'eux-mêmes régi uniquement par leurs conditions de bien-être individuel. Dès lors, ces accompagnés deviennent des accompagnants, et ainsi le gouvernement des autres qu'ils peuvent procurer renforce leur propre gouvernement d'eux-mêmes vers une subjectivation éthique, puisqu'ils mettent en œuvre les principes de solidarité qui les réunissent.

Cet exemple montre qu'il est aujourd'hui possible de mettre en place des interventions éducatives en santé publique qui évitent les affres de la biopolitique au profit d'un maintien de la subjectivation éthique des acteurs de la relation éducative. L'apprentissage par accompagnement de la constitution d'une relation de soi à soi telle qu'elle favorise la création de normes individuelles est un modèle pour l'éducation en santé.

\section{Vers une éducation à la santé comme éducation au corps}

On peut ainsi penser l'éducation en santé comme une éducation au corps, un apprentissage des modalités de vie avec son corps, de vie en son corps, en tant qu'il est impossible de distinguer, dans le vécu individuel, le sujet de sa corporéité. L'éducation au corps, qui remplacerait l'éducation du corps, viserait donc le développement d'un rapport sain de soi à soi (et donc de soi aux autres) du sujet corporel, autrement dit le développement d'une conscience du corps (Shusterman, 2007) apte à développer les potentialités du sujet en matière de santé. Cela répondrait mieux aux exigences de la santé qui est, comme on le sait depuis Canguilhem (1988), moins un concept scientifique permettant une transmission pédagogique théorique, qu'une notion vulgaire nécessitant une praxis de son propre corps afin d'en intégrer les normes toujours subjectives. L'éducation au corps consiste donc en l'acquisition de ce que Foucault nomme, dans sa relecture des textes antiques, une paraskeuê, c'est-à-dire, un ensemble de dispositions incorporées et ainsi toujours disponible pour faire face aux modifications de l'existence issues de la relation du sujet corporel à son environnement. L'éducateur en santé doit se soucier du souci que celui qu'il souhaite éduquer a de lui-même et donc de son corps. Son action doit donc prendre la forme du développement de ce souci de soi, de son corps et par conséquent de sa santé. C'est en ce sens qu'il peut s'affirmer comme un véritable patricien réflexif (Schön, 1994) constituant son action éducative au fur et à mesure que se construit la relation éducative, seule alternative à l'imposition biopolitique de normes préconçues. Mais pour cela, l'éducateur en santé doit faire à son tour preuve de liberté en transgressant les normes de l'éducation en santé actuelle.

Ainsi convient-il de reconnaître l'identité de l'éducateur en santé, en lui fournissant un statut professionnel tout à la fois proche des personnels médicaux, et néanmoins distinct. Ce personnage tiers, véritable praticien-chercheur (Delavergne, 2007), pourrait ainsi 
permettre de faire le lien entre un monde médical qui peine, malgré les apports récents dans sa formation des sciences humaines et sociales, à sortir d'une rationalité biomédicale proprement instrumentale, et un monde de l'éducation souvent submergé par ses propres objectifs et qui supporterait mal la multiplication de ses tâches. Dès lors, serait-il possible d'envisager, dans comme hors de l'école [15], la multiplication de véritables interventions éducatives de santé, à l'instar de ce que réalise par exemple EPIDAURE, département prévention du Centre Régional de Lutte contre le Cancer de Montpellier, qui a mis en place, sur le thème de la nutrition, un dispositif ludo-pédagogique à vocation d'éducation pour la santé [16]. Dans un cadre ni médical, ni proprement scolaire, des enfants de 3 à 12 ans peuvent ainsi, par l'intermédiaire de dispositifs avant tout ludiques, acquérir des savoirs sur les aliments et l'équilibre alimentaire mais également développer des compétences psycho-sociales quant à leur propre alimentation, et ce dans le but, de les voir adopter des attitudes et comportements favorables à son développement harmonieux. Dans une thématique similaire, le projet Nutrisport (Mesurolle, 2009), porté par la MGEN et organisé au sein de l'université du Littoral Côte d'Opale (site de Boulogne sur Mer), a permis de favoriser l'adaptation des habitudes alimentaires des personnels éducatifs à leur dépenses énergétiques par le biais d'actions tant formatives qu'informatives. Outre les séances d'informations mises en place par les éducateurs en santé de la MGEN, le programme proposait des ateliers de cuisine, des randonnées pédestres, l'organisation de repas communs, etc. ; autrement dit, un espace où pratique et théorie étaient étroitement associées afin de conduire, dans un échange des savoirs et des compétences, les personnels universitaires à se soucier de leur santé et de leur alimentation. Enfin, le projet «gymkhana » mené, en 2009, par Nicolas Callewaert dans la maison de retraite «Le Clos des Tilleuls» se présente comme un exemple pertinent de l'éducation au corps que nous revendiquons. Cette intervention éducative vise à développer, chez les personnes âgées prothésées, l'apprentissage de nouveaux gestes, de nouvelles attitudes, postures et positions nécessaires au maintien et à l'accroissement de leur autonomie, tout en insistant sur la nécessaire recalibartion de leur image corporelle à l'aune des modifications engendrées par la prothèse. Par le biais d'une approche pluridisciplinaire où l'éducateur en santé fait le lien entre une psychomotricienne, des aides-soignantes et infirmières et un animateur sportif, un travail est mené sur les déplacements des personnes âgées à l'intérieur et à l'extérieur de la structure, autour de parcours spécifiques visant l'entraînement corporel, autant que l'engagement d'une réflexion sur les aménagements environnementaux qui favoriseraient la mobilité (Callewaert, 2009).

Des initiatives de ce type, organisées par des éducateurs en santé, se multiplient aujourd'hui en France, faisant écho à l'accroissement considérable des recherches universitaires menées dans ce domaine et dont témoignent les nombreux congrès [17], colloques [18], séminaires [19], thèses [20] et publications actuelles [21]. L'éducation en

15 Pour rependre le titre du dernier congrès du Réseau des IUFM pour la formation en éducation à la santé et prévention des conduites addictives qui s'est tenu à Paris les 21 et 22 octobre 2010.

16 Voir à ce propos l'intervention de Pauline Thabuteau et Jean-Christophe Azorin, intitulée L'éducation pour la santé à l'école : la question de la cohérence des démarches, méthodes et outils, lors du symposium Education et santé du dernier congrès de l'AREF à Genève en septembre 2010.

17 Nous pensons ici notamment au congrès de l'AREF qui s'est tenu à Genève en septembre 2010, et qui comptait quatre symposiums consacrés à l'éducation en santé mais également au congrès du Réseau des IUFM pour la formation en éducation à la santé et prévention des conduites addictives qui a eu lieu à Paris les 21 et 22 octobre 2010 .

18 Nous pensons ici au colloque «Bien-être ou être bien ?» qui s'est déroulé à Nancy le 4 juin 2010 et qui interrogeait la formation des éducateurs en santé autour des techniques douces.

19 Le séminaire d'Histoire, d'épistémologie et de philosophie des sciences du corps dirigé par B. Andrieu a abordé en 2010-2011 le problème de l'éducation corporelle. 
santé s'affirme ainsi, jour après jour, comme un champ de recherche à part entière et un domaine de pratiques nécessaire dans une société pour qui le corps comme la santé est un enjeu majeur (Halpern, 2010).

Alexandre Klein, LHSP Archives H. Poincaré / UMR 7117 CNRS / Nancy Université

\section{Bibliographie}

- Andrieu B. (2009). La philosophie du corps. Paris : Vrin.

- Andrieu B. (2004). La fin de la biopolitique chez Michel Foucault. Le Portique [En ligne] http://leportique.revues.org/index627.html. (consulté le 15 septembre 2009)

- Andrieu B. (2001). La nouvelle philosophie du corps. Ramonville Saint-Agne : Erès.

- Barroux G. (2008) Philosophie, maladie et médecine au XVIII siècle. Paris : Honoré Champion.

- Berlivet L. (2004). Une biopolitique de l'éducation pour la santé. In D. Memmi et D. Fassin, Le gouvernement des corps. Paris : EHESS, p. 37-75.

- Bohuon A. (inédit). La pratique physique et sportive féminine à la fin du XIX ${ }^{\mathrm{e}}$ siècle : entre vertus thérapeutiques et/ou pathogènes sur les organes génitaux.

- Brohm J.-M. (2001). Le corps analyseur. Paris : Economica.

- Bruaire C. (1968). Philosophie du corps. Paris : Seuil.

- Canguilhem G. (1963). Le normal et le pathologique. Paris : PUF.

- Canguilhem G. (1988). La santé : concept vulgaire et question philosophique. Conférence donnée à Strasbourg en mai 1988 à l'invitation du Pr Lucien Braun. Cahiers du séminaire de philosophie $n^{\circ} 8$ : la santé. Strasbourg: Editions Centre de documentation en histoire de la philosophie, p. 119-133.

- Callewaert N. (2009). Le corps hybride de la personne âgée, pour une nouvelle approche de la prévention des chutes en maison de retraite. Mémoire de Master 2 «Education et santé », UFR Sciences de l'éducation, Université Lille 3, sous la direction de B. Andrieu et J. Descarpentries.

- Dedeine M. (2009). De l'organisme communautaire à la santé individuelle. En quoi l'approche communautaire peut amener l'individu au gouvernement de soi. Mémoire de Master 2 «Education et santé », UFR Sciences de l'éducation, Université Lille 3, sous la direction de J. Descarpentries et d'A. Klein.

- Delaporte F. (1990). Le savoir de la maladie. Paris : PUF.

- De Lavergne C. (2007). La posture du praticien-chercheur: un analyseur de l'évolution de la recherche qualitative. Recherches qualitatives, Hors-série, $\mathrm{n}^{\circ} 3$, Actes du colloque Bilan et prospectives de la recherche qualitative, p. 28-42.

- Foucault M. (2008). Le gouvernement de soi et des autres. Cours au collège de France 1982-1983. Paris : Gallimard Seuil.

20 Par exemple, la thèse de Carine Simar, « Analyse de l'activité des enseignants du premier degré en éducation à la santé : de la tâche prescrite à la tâche représentée : étude de leur travail en classe et hors classe », soutenue le 9 décembre 2010 à Clermont-Ferrand sous la direction de Didier Jourdan.

21 Par exemple, Recherches \& éducations, $\mathrm{n}^{\circ}$ 3, "Santé et éducation », 2010, sous la direction de J. Descarpentries, A. Klein, S. Parayre, ou la revue Spirale qui prépare actuellement un numéro consacré à l'éducation et la santé, et bien sûr le présent volume. 
- Foucault M. (2004). Naissance de la biopolitique. Cours au Collège de France 19781979. Paris : Gallimard Seuil.

- Foucault M. (2001). L'éthique du souci de soi comme pratique de la liberté (entretien avec H. Becker, R. Fornet-Betancourt, A. Gomez-Müller, 20 janvier 1984). Concordia. Revista internacional de filosofia, $\mathrm{n}^{\circ} 6$, juillet-décembre 1984, p. 99-116, repris dans Dits et écrits. Paris : Quarto Gallimard, 2001, vol. 2, texte 356, p. 1527-1548.

- Foucault M. (1997). Il faut défendre la société, Cours au Collège de France 19751976. Paris : Gallimard-Seuil.

- Foucault M. (1984a). Histoire de la sexualité 2 (l'usage des plaisirs). Paris : Gallimard.

- Foucault M. (1984b). Histoire de la sexualité 3 (le souci de soi). Paris : Gallimard.

- Foucault M. (1976). Histoire de la sexualité 1 (la volonté de savoir). Paris : Gallimard.

- Foucault M. (1975). Surveiller et punir. Paris : Gallimard.

- Frank J.-P. (1779-1819). Système complet de police médicale. Manheim.

- Gayon J. et Jacobi D. (2006). L'éternel retour de l'eugénisme. Paris : PUF.

- Halpern C. (2010). La Santé, enjeu humain, enjeu de société. Paris : Editions Sciences Humaines.

- Han B. (1998). L'ontologie manquée de M. Foucault. Grenoble : Jerome Million.

- Herzlich C. (1969). Santé et maladie. Paris : EHESS.

- Illich I. (1975). Némésis médicale. Paris : Seuil.

- Klein A. (2010a). Les sensations de santé. Nancy : P.U. Nancy.

- Klein A. (2010b). Quel sujet pour l'éducation à la santé ? Les apports de Michel Foucault pour les interventions éducatives en santé publique. Recherches \& éducations, $\mathrm{n}^{\circ}$ 3, Santé et éducation, p. 47-75.

- Klein, A. (2008). La santé comme norme de soin. Philosophia Scientice, 12(2), p. 213227.

- Kuhn T. (1962). La structure des révolutions scientifiques. Paris : Champ Flammarion, 1983.

- Le Blanc G. (2002). La Vie Humaine ; Anthropologie et Biologie Chez Georges Canguilhem. Paris : PUF.

- Legrand S. (2007). Les normes chez Foucault. Paris : PUF.

- Massé R. (2003). Ethique et santé publique. Laval : PUL.

- Mesurolle C. (2009). Prévention et rapport au corps. Mémoire de Master 2 «Education et santé », UFR Sciences de l'éducation, Université Lille 3, sous la direction de F. Danvers et A. Klein.

- O'Followell L. (1900). Bicyclette et organes génitaux. Paris : J-B Baillière et fils.

- Parayre S. (2009). L'hygiène à l'école aux XVIIe et XIXe siècles. Recherches \& éducations, $\mathrm{n}^{\circ} 1$, p. 177-193.

- Pichot A. (2000). La Société pure : de Darwin à Hitler. Paris : Flammarion.

- Revel J. (2004). Michel Foucault: discontinuité de la pensée ou pensée du discontinu ?. Le Portique [En ligne] http://leportique.revues.org/index635.html. (consulté le 15 septembre 2009).

- Schön D. (1994). Le Praticien Réflexif : à la recherche du savoir caché dans l'agir des professionnels. Montréal : Les éditions logiques.

- Shusterman R. (2007). Conscience du corps :pour une soma-esthétique. Paris: Editions de l'Eclat.

- Stengers I. (1998). La guerre des sciences : et la paix ?. Alliages, 1998, (p.35-36).

- Szasz T. (1975). Le mythe de la maladie mentale. Paris : Payot.

- Tessier S., Andreys J.-B. et Ribeiro, M.-A. (1996). Santé publique et santé communautaire. Paris : Maloine. 
- Tissot S.-A. (1760). L'onanisme. Lausanne : Grasset,

- Veyne P. (2008). Foucault, sa pensée, sa personne. Paris : Albin Michel. 